\title{
Evaluation of the Manchester triage system for patients with acute coronary syndrome
}

\author{
Daniel Kiblboeck · Klara Steinrueck - Christian Nitsche · Wolfgang Lang • Joerg Kellermair • \\ Hermann Blessberger · Clemens Steinwender · Peter Siostrzonek
}

Received: 7 October 2019 / Accepted: 4 March 2020 / Published online: 2 April 2020

(C) The Author(s) 2020

\begin{abstract}
Summary
Background An early diagnosis of acute coronary syndrome (ACS) is crucial for treatment and prognosis. The aim of this study was to evaluate the Manchester triage system (MTS) for patients with ACS, e.g. ST-segment elevation myocardial infarction (STEMI), nonST elevation myocardial infarction (N-STEMI) and unstable angina pectoris (UAP).

Methods Retrospective analysis of patients diagnosed with ACS (STEMI, N-STEMI and UAP) who were triaged in the emergency department (ED) with the MTS.

Results In this study 282 patients with ACS (STEMI: $34.0 \%$, N-STEMI: $61.7 \%$, UAP: $4.3 \%$ ) were triaged as MTS level 1 (immediate assessment): $0.4 \%$, MTS level 2 (very urgent): $51.4 \%$, MTS level 3 (urgent): $41.5 \%$, MTS level 4 (standard): $6.7 \%$, MTS level 5 (non-urgent): $0 \%$. We observed significantly lower mean MTS levels in males (male: $2.48 \pm 0.59$, female: $2.68 \pm 0.68, p=0.02)$ and in patients younger than 80 years (age $<80$ years: $2.50 \pm 0.61$, age $\geq 80$ years: $2.70 \pm 0.67, p=0.03$ ). We did not find a significant difference of mean MTS levels in different types of ACS (STEMI: $2.46 \pm 0.6$, N-STEMI: $2.59 \pm 0.64$, STEMI
\end{abstract}

\footnotetext{
D. Kiblboeck, M.D. $(\bowtie) \cdot$ J. Kellermair · H. Blessberger · C. Steinwender

Department of Cardiology and Medical Intensive Care, Med Campus III, Kepler University Hospital, Medical Faculty of Johannes Kepler University, Krankenhausstraße 9, 4021 Linz, Austria

daniel.kiblboeck@kepleruniklinikum.at

K. Steinrueck · W. Lang · P. Siostrzonek

Department of Internal Medicine II - Cardiology,

Krankenhaus der Barmherzigen Schwestern, Linz, Austria

\section{Nitsche}

Department of Internal Medicine II, Medical University of Vienna, Vienna, Austria
}

VS N-STEMI: $p=0.11$, UAP: $2.67 \pm 0.65$, STEMI vs UAP: $p=0.26$ ) and with respect to diabetes (diabetic: $2.47 \pm 0.57$, non-diabetic: $2.58 \pm 0.65, p=0.13$ ). The inhospital mortality was $2.5 \%$ (MTS level 2: $n=3$, MTS level 3: $n=3$, MTS level 4: $n=1$ ).

Conclusion The majority of patients with ACS were classified as MTS levels 2 and 3. There was no significant difference of mean MTS levels in patients with STEMI, NSTEMI and UAP. In order to assure an early diagnosis of STEMI, an electrocardiogram (ECG) should be carried out immediately or at least within 10 min after first medical contact in the ED in all patients suspected for ACS, irrespective of the assigned MTS level.

Keywords Manchester triage system · Emergency medicine $\cdot$ Coronary artery disease $\cdot$ Myocardial infarction - Chest pain

\section{Introduction}

Chest pain is a frequent complaint in the emergency department (ED). Approximately $5-20 \%$ of all ED admissions present with chest pain [1]; however, the diagnosis of acute coronary syndrome (ACS) is only confirmed in about $10 \%$ of patients who present with chest pain in the ED [2, 3]. An early diagnosis of ACS particularly in patients with ST-elevation myocardial infarction (STEMI), is crucial for treatment and prognosis.

The Manchester triage system (MTS), which was developed by the Manchester Triage Group in 1994, classifies patients based on their main symptoms into five different levels of urgency in terms of the need for first medical assessment ('MTS level $1=$ immediate assessment', 'MTS level 2=very urgent assessment within 10 min', 'MTS level $3=$ urgent assessment within $30 \mathrm{~min}$ [German version]/60 min [British version]', 
'MTS level 4=standard assessment within 90 min [German version]/120 min [British version]', 'MTS level $5=$ non-urgent assessment within $120 \mathrm{~min}$ [German version] $/ 240 \mathrm{~min}$ [British version]'), irrespective of the eventual diagnosis [4]. Therefore, the MTS uses 52 different flow chart diagrams, e.g. chest pain, abdominal pain, with defined key discriminators, such as danger to life, respiratory distress or state of consciousness to determine the level of urgency for assessment [4].

There is limited data whether there is an influence of MTS levels on treatment strategies and outcome parameters in patients with ACS. The aim of this study was to evaluate the MTS for patients diagnosed with ACS who present primarily in the ED.

\section{Methods}

Patients were identified retrospectively for eligibility in this study by International Statistical Classification of Diseases (ICD) tracking for ICD diagnosis I21.0 (acute transmural myocardial infarction of anterior wall), ICD I21.1 (acute transmural myocardial infarction of inferior wall), I21.2 (acute transmural myocardial infarction of other sites), I21.3 (acute transmural myocardial infarction of unspecified site), I21.4 (acute subendocardial myocardial infarction), I21.9 (acute myocardial infarction, unspecified). All patients with a confirmed diagnosis of ACS (STEMI, non-ST elevation myocardial infarction (N-STEMI) or unstable angina pectoris (UAP), who presented primarily in the ED over a study period of 12 months, were enrolled in this retrospective, single center study. The study was conducted at a large general hospital with an ED volume of about 33,000 patient visits per year with a 24/7 cardiac catheterization laboratory onsite. The triage was done by experienced emergency nurses who had attended an education program for the MTS. Data were collected by retrospective chart review.

The primary objective of this study was defined as the distribution of different MTS levels in patients with ACS. The secondary objectives were defined as a prespecified subgroup analysis of the MTS level distribution for gender, diabetic patients, different types of ACS (STEMI, N-STEMI and UAP) and age younger and older than 80 years. The study design was approved by the local ethics committee and the study was conducted according to the Declaration of Helsinki.

\section{Statistical analysis}

Categorical variables are described as absolute numbers and percentages. Continuous values are presented as means \pm standard deviation. Differences between groups involving normally distributed data were analyzed by the unpaired t-test; those involving not normally distributed data, by the Mann-Whitney $\mathrm{U}$ test; and those involving proportions, by the $\chi^{2}$-test.
A two-sided $p$-value $<0.05$ was considered statistically significant. The $p$-value was not adjusted for multiple testing because the retrospective design of this study was only suitable for hypothesis-generating results. All calculations were performed with SPSS statistical software (Version 22, SPSS Inc., Chicago, IL, USA).

\section{Results}

During the study period, 431 patients were treated for ACS at this cardiac care unit, 282 of these patients $(68.3 \%)$ presented primarily in the ED and were triaged with the MTS (Fig. 1). The mean age was $68.0 \pm 14.6$ years and 90 patients $(31.9 \%)$ were female. Ninety-six patients (34.0\%) presented with STEMI and underwent immediate revascularization in the coronary catheterization laboratory: 174 patients $(61.7 \%)$ were diagnosed as N-STEMI and 12 patients (4.3\%) as UAP. Coronary angiography was performed in 259 patients $(91.8 \%)$, while 23 patients $(8.2 \%)$ were treated conservatively due to severe comorbidities. Overall, 216 patients $(76.8 \%)$ required percutaneous coronary intervention (STEMI: $n=81,84.4 \%$, N-STEMI: $n=130$, $74.7 \%$ ) and 3 patients were scheduled for coronary artery bypass surgery. The demographic and clinical characteristics of the study population are presented in Table 1.

The primary objective, which was the distribution of MTS levels in patients with ACS, was $0.4 \%$ $(n=1)$ for MTS level 1, 51.4\% $(n=145)$ for MTS level 2, 41.5\% ( $n=117)$ for MTS level 3, 6.7\% $(n=19)$ for MTS level 4 and $0 \%$ for MTS level 5 (Fig. 2). While 195 patients $(69.1 \%)$ presented with the chief complaint chest pain (STEMI: $n=76,80.0 \%$, N-STEMI: $n=109$, $63.4 \%$, UAP: $n=10,83.3 \%$ ), 87 patients had other symptoms. Overall, shortness of breath was the second most frequently used flow chart diagram (all: $n=32,11.3 \%)$, STEMI: $n=5,5.3 \%$, N-STEMI: $n=24$, $14.0 \%$, UAP: $n=2,16.7 \%$ followed by the flow chart diagrams unwell adult (all: $n=24,8.5 \%$, STEMI: $n=5$, 5.3\%, N-STEMI: $n=17,9.9 \%$ ), palpitations (all: $n=8$, 2.8\%, STEMI: $n=1,1.1 \%, \mathrm{~N}-\mathrm{STEMI}: n=7,4.1 \%)$ and

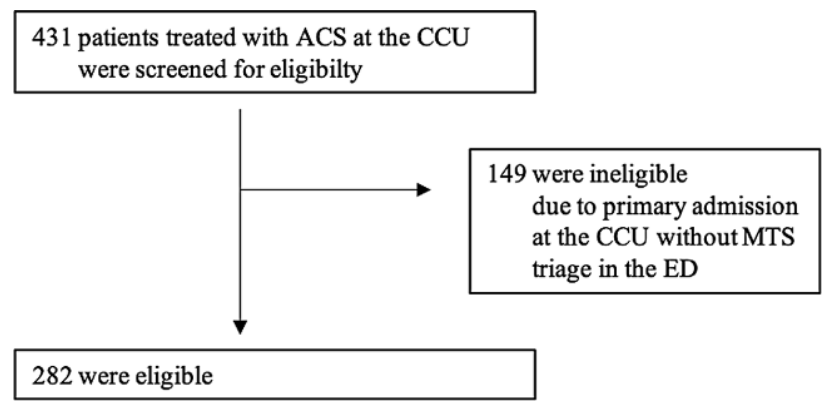

Fig. 1 Screening for eligibility in the study. ACS acute coronary syndrome; STEMI ST-elevation myocardial infarction, $N$-STEMI non ST-elevation myocardial infarction; UAP unstable angina pectoris; CCU cardiac care unit; MTS Manchester Triage System 
Table 1 Demographic and clinical characteristics of the study population

\begin{tabular}{|c|c|c|c|c|}
\hline & $\begin{array}{l}\text { All } \\
(n=282)\end{array}$ & $\begin{array}{l}\text { STEMI } \\
(n=96)\end{array}$ & $\begin{array}{l}\text { N-STEMI } \\
(n=174)\end{array}$ & $\begin{array}{l}\text { UAP } \\
(n=12)\end{array}$ \\
\hline Age, years $\pm S D$ & $68.0 \pm 14.6$ & $66.2 \pm 15.6$ & $69.4 \pm 13.9$ & $61.8 \pm 15.5$ \\
\hline Age $\geq 80$ years, $n(\%)$ & $61(21.6)$ & $22(22.9)$ & 38 (21.8) & $1(8.3)$ \\
\hline Female, $n(\%)$ & $90(31.9)$ & 37 (38.5) & $51(29.3)$ & $2(16.7)$ \\
\hline Hypertension, $n(\%)$ & 197 (69.9) & $56(58.3)$ & $132(75.9)$ & $9(75.0)$ \\
\hline Diabetes, $n(\%)$ & $90(31.9)$ & $24(25.0)$ & $60(34.5)$ & $6(50.0)$ \\
\hline Smokers, $n(\%)$ & $76(27.0)$ & $27(28.1)$ & 45 (25.9) & $4(33.3)$ \\
\hline Hyperlipidemia, $n(\%)$ & $170(60.3)$ & $52(54.2)$ & $107(61.5)$ & 11 (91.7) \\
\hline Prior STEMI, $n(\%)$ & $41(14.5)$ & $5(5.2)$ & $30(17.2)$ & $6(50.0)$ \\
\hline Prior PCl, $n(\%)$ & $58(20.6)$ & $11(11.5)$ & $41(23.6)$ & $6(50.0)$ \\
\hline Prior CABG, $n(\%)$ & $8(2.8)$ & $1(1.0)$ & $7(4.0)$ & $0(0)$ \\
\hline Cardiogenic shock, $n(\%)$ & $7(2.5)$ & $5(5.2)$ & $2(1.1)$ & $0(0)$ \\
\hline $\mathrm{PCl}, n(\%)$ & $216(76.8)$ & $81(84.4)$ & $130(74.7)$ & $5(41.7)$ \\
\hline CABG, $n(\%)$ & $3(1.1)$ & $0(0)$ & $3(1.7)$ & $0(0)$ \\
\hline Acetylsalicylic acid, $n(\%)$ & $276(98.2)$ & $96(100)$ & $168(96.6)$ & $12(100)$ \\
\hline Clopidogrel, $n(\%)$ & $71(25.2)$ & $16(16.7)$ & $48(27.6)$ & $7(58.3)$ \\
\hline Prasugrel, $n(\%)$ & $59(20.9)$ & $51(53.1)$ & $7(4.0)$ & $1(8.3)$ \\
\hline Ticagrelor, $n(\%)$ & $148(52.5)$ & $31(32.3)$ & $113(64.9)$ & $4(33.3)$ \\
\hline In-hospital mortality, $n$ (\%) & $7(2.5)$ & $2(2.1)$ & $5(2.9)$ & $0(0)$ \\
\hline
\end{tabular}

collapsed adult (all: $n=8,2.8 \%$; STEMI: $n=2,2.1 \%$; N-STEMI: $n=5,2.9 \%$ ) (Fig. 2).

We observed a significant difference between mean MTS levels with respect to gender (male: $2.48 \pm 0.59$, female: $2.68 \pm 0.68, p=0.02$ ) and age (age $<80$ years: $2.50 \pm 0.61$, age $\geq 80$ years: $2.70 \pm 0.67, p=0.03$ ). There was no significant difference in different types of ACS (STEMI: $2.46 \pm 0.6$, N-STEMI: $2.59 \pm 0.64$, STEMI vs. N-STEMI: $p=0.11$, UAP: $2.67 \pm 0.65$, STEMI vs. UAP: $p=0.26$ ) and with respect to diabetes (diabetic: $2.47 \pm 0.57$, non-diabetic: $2.58 \pm 0.65, p=0.13$; Table 2).

The documented time of the first ECG was available in 168 patients of our study population. The mean

Table 2 Mean MTS levels and standard deviation of the study population and in pre-specified subgroups (different types of acute coronary syndrome, male vs female patients and patients with diabetes vs without diabetes)

\begin{tabular}{|l|l|l|}
\hline & Mean MTS level \pm SD & $p$-value \\
\hline All & $2.55 \pm 0.63$ & - \\
\hline STEMI & $2.46 \pm 0.6$ & - \\
\hline N-STEMI & $2.59 \pm 0.64$ & 0.11 \\
\hline UAP & $2.57 \pm 0.65$ & 0.26 \\
\hline Male & $2.48 \pm 0.59$ & - \\
\hline Female & $2.68 \pm 0.68$ & 0.02 \\
\hline Age $<80$ years & $2.50 \pm 0.61$ & - \\
\hline Age $\geq 80$ years & $2.70 \pm 0.67$ & 0.03 \\
\hline Diabetes & $2.47 \pm 0.57$ & - \\
\hline No diabetes & $2.58 \pm 0.65$ & 0.13 \\
\hline $\begin{array}{l}M T S \text { Manchester Triage System; STEMIST-elevation myocardial infarction; } \\
\text { N-STEMI non ST-elevation myocardial infarction; UAP unstable angina } \\
\text { pectoris }\end{array}$
\end{tabular}

time interval from MTS to ECG was $13.5 \pm 17.8 \mathrm{~min}$. There was a shorter mean time interval from MTS to ECG for STEMI patients than for N-STEMI patients (STEMI: $n=62,9.5 \pm 14.2 \mathrm{~min}$, N-STEMI: $n=98$, $16.7 \pm 19.8 \mathrm{~min})$. The mean time intervals from MTS to ECG were $12 \mathrm{~min}$ for MTS level $1(n=1), 9.0 \pm 14.6 \mathrm{~min}$ for MTS level $2(n=89), 19.5 \pm 20.8 \mathrm{~min}$ for MTS level 3 $(n=69), 11.3 \pm 8.0 \mathrm{~min}$ for MTS level $4(n=9)$. Overall, the first ECG was recorded in 115 of the 168 patients $(68.5 \%)$ within $10 \mathrm{~min}$ after MTS (STEMI, $n=62: 49$ patients [79.0\%], N-STEMI, $n=98$ : 58 patients $[59.2 \%])$. The mean maximum values of creatinine kinase as a marker of infarct size were for patients with STEMI or N-STEMI 1707U/l (MTS level 1), $891 \pm 1229 \mathrm{U} / \mathrm{l}$ (MTS level 2), $889 \pm 1436 \mathrm{U} / \mathrm{l}$ (MTS level 3), and 660 $\pm 1157 \mathrm{U} / \mathrm{l}$ (MTS level 4).

Overall, seven patients had cardiogenic shock at the CCU, two of these presented with cardiogenic shock at the time of triage, five developed this subsequently. Of these patients one was triaged as MTS level 1, four patients as MTS level 2 and two patients as MTS level 4 (Table 3$)$. The in-hospital mortality was $2.5 \%(n=7)$, three patients of these patients were triaged as MTS level 2 and 3, respectively and one patient was assigned to MTS level 4 .

\section{Discussion}

Our retrospective study demonstrates that the majority of patients $(92.9 \%)$ diagnosed with ACS who presented primarily in the ED were triaged as MTS level 2 and MTS level 3 (very urgent to urgent assessment within 10-30 min [German version] or $60 \mathrm{~min}$ [British 
Fig. 2 Manchester Triage System (MTS) results of the study population [\%]: blue columns $=$ all $(n=282)$; orange columns $=$ STEMI $(n=95)$; grey columns $=$ N-STEMI $(n=172)$ and yellow columns $=\operatorname{UAP}(n=12)$. ACS acute coronary syndrome; STEMI ST-elevation myocardial infarction; $N$-STEMI non ST-elevation myocardial infarction; UAP unstable angina pectoris

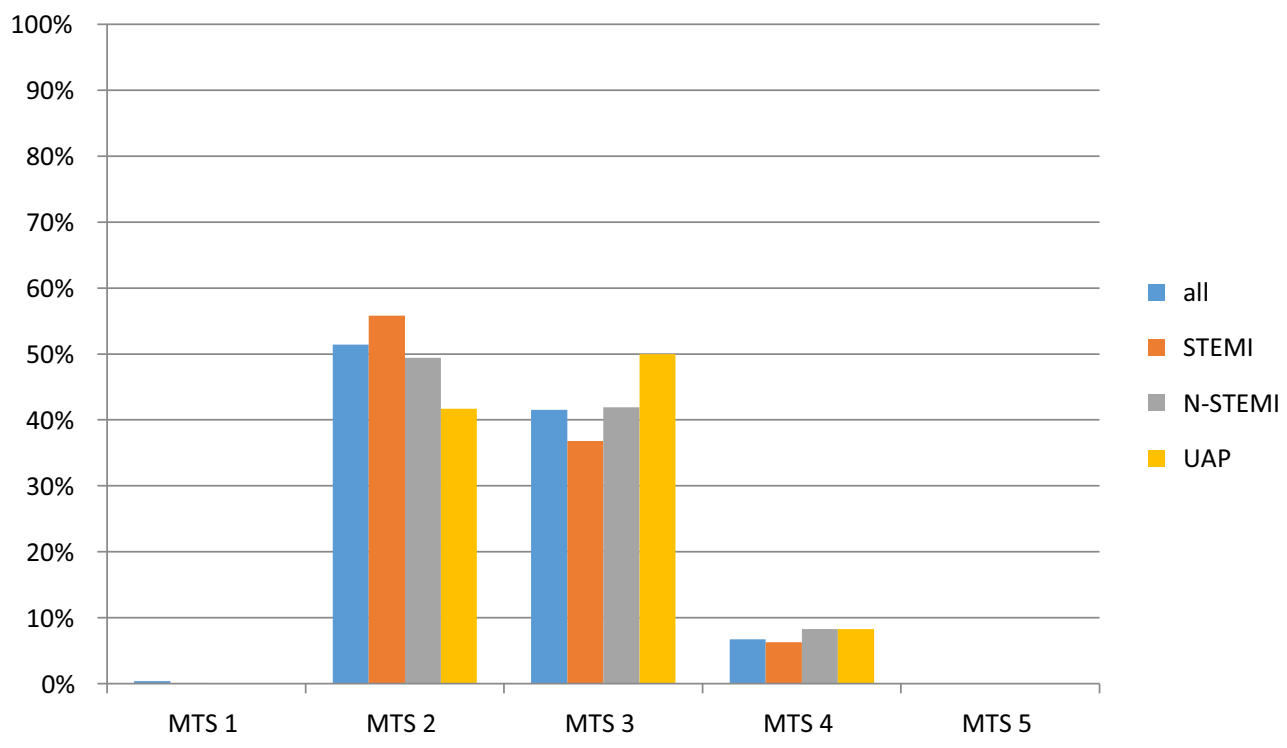

Table 3 Triage results of patients with cardiogenic shock. One patient was triaged as MTS level 1, 4 patients as MTS level 2 and 2 patients as MTS levels 4 . Shortness of breath was the most frequently used MTS flowchart. Five of these patients with STEMI had a cardiogenic shock. Overall, 5 patients developed cardiogenic shock after MTS in the ED. The in-hospital mortality of patients with cardiogenic shock was $28.6 \%(n=2)$

\begin{tabular}{|c|c|c|c|c|c|c|}
\hline ID & MTS level & MTS flow chart & BP at MTS & Type of ACS & Shock onset & Status at hospital discharge \\
\hline ID28 & 2 & Shortness of breath & $105 / 71$ & N-STEMI & After MTS & Alive \\
\hline ID103 & 4 & Shortness of breath & $163 / 80$ & N-STEMI & After MTS & Deceased \\
\hline ID124 & 1 & Shortness of breath & $87 / 49$ & STEMI & Before MTS & Alive \\
\hline ID169 & 2 & Chest pain & $108 / 62$ & STEMI & After MTS & Alive \\
\hline ID317 & 2 & Collapsed adult & $61 / 32$ & STEMI & Before MTS & Alive \\
\hline ID327 & 2 & Shortness of breath & $119 / 97$ & STEMI & After MTS & Deceased \\
\hline ID413 & 4 & Vomiting & $134 / 66$ & STEMI & After MTS & Alive \\
\hline
\end{tabular}

version]). We observed significantly lower MTS levels for males and patients younger than 80 years while there was no significant difference with respect to different types of ACS and patients with diabetes.

Due to an increasing number of patient visits in ED, triage tools become more important in ED to prioritize the assessment and treatment of patients with potentially life-threatening diseases, such as ACS. An early diagnosis of STEMI in the ED is crucial for an early revascularization strategy in the coronary catheterization laboratory.

Patients who present to the ED are classified by the MTS based on their chief complaints into five different levels of urgency for their need of assessment according to 52 different flowchart diagrams, irrespective of the eventual diagnosis [4].

The recommended target time for an ECG is less than $10 \mathrm{~min}$ after first medical contact for patients with ACS according to current guidelines in our study population [5]. Data about the documented time of the first ECG after MTS was available in 168 of our study population. Therefore, the presented results need to be interpreted with caution. However, we detected a shorter mean time interval from MTS to the first ECG for STEMI patients compared to N-STEMI patients (STEMI: $9.5 \pm 14.2 \mathrm{~min}, \mathrm{~N}$-STEMI: $16.7 \pm 19.8 \mathrm{~min})$. Remarkably, we observed a trend for mean time intervals from MTS to the first ECG MTS with respect to different MTS levels (MTS $1(n=1)$ : $12 \mathrm{~min}$, MTS $2(n=89): 9.0 \pm 14.6 \mathrm{~min}$, MTS $3(n=69)$ : $19.5 \pm 20.8 \mathrm{~min}$, MTS $4(n=9): 11.3 \pm 8.0 \mathrm{~min})$. The first ECG was written in $68.5 \%(n=115)$ of patients with ACS, $79.0 \%$ of STEMI patients $(n=49)$ and $59.2 \%$ of NSTEMI patients $(n=58)$ within $10 \mathrm{~min}$ after MTS. Gouvea et al. reported a mean time interval from MTS to ECG for all patients with ACS of $24.9 \pm 31.1 \mathrm{~min}$ and a mean time interval from MTS to ECG for MTS level 1 and 2 patients of $19.46 \pm 24.62 \mathrm{~min}$ [6]. Therefore, we consider that in patients with suspected ACS it is very important to write an ECG immediately or at least within $10 \mathrm{~min}$ after first medical contact in the ED irrespective of the assigned MTS level, in order to assure an early diagnosis of STEMI in this high-risk population. Short door-to-balloon times are important for prognosis in patients with STEMI. We observed a trend for higher mean maximum val- 
Fig. 3 Flow chart diagrams used for patients with ACS: blue columns = 'all $(n=282)$ ', orange columns = 'STEMI $(n=95)$ ', $\quad$ grey columns $=$ 'N-STEMI $(n=172)$ ' and yellow columns $=$ 'UAP $(n=12)^{\prime}$. Chest pain was the most frequently used flowchart diagram (all: $69.1 \%$, STEMI: $80.0 \%$, N-STEMI: $63.4 \%$, UAP: $83.3 \%$ ) followed by shortness of breath in adults (all: $11.3 \%$, STEMI: 5.3\%, N-STEMI: $14.0 \%$, UAP: $16.7 \%$ ) and unwell adult (all: 8.9\%, STEMI: $5.3 \%$, N-STEMI: 9.9\%). ACS acute coronary syndrome; STEMI STelevation myocardial infarction; $N$-STEMI non STelevation myocardial infarction; UAP unstable angina pectoris

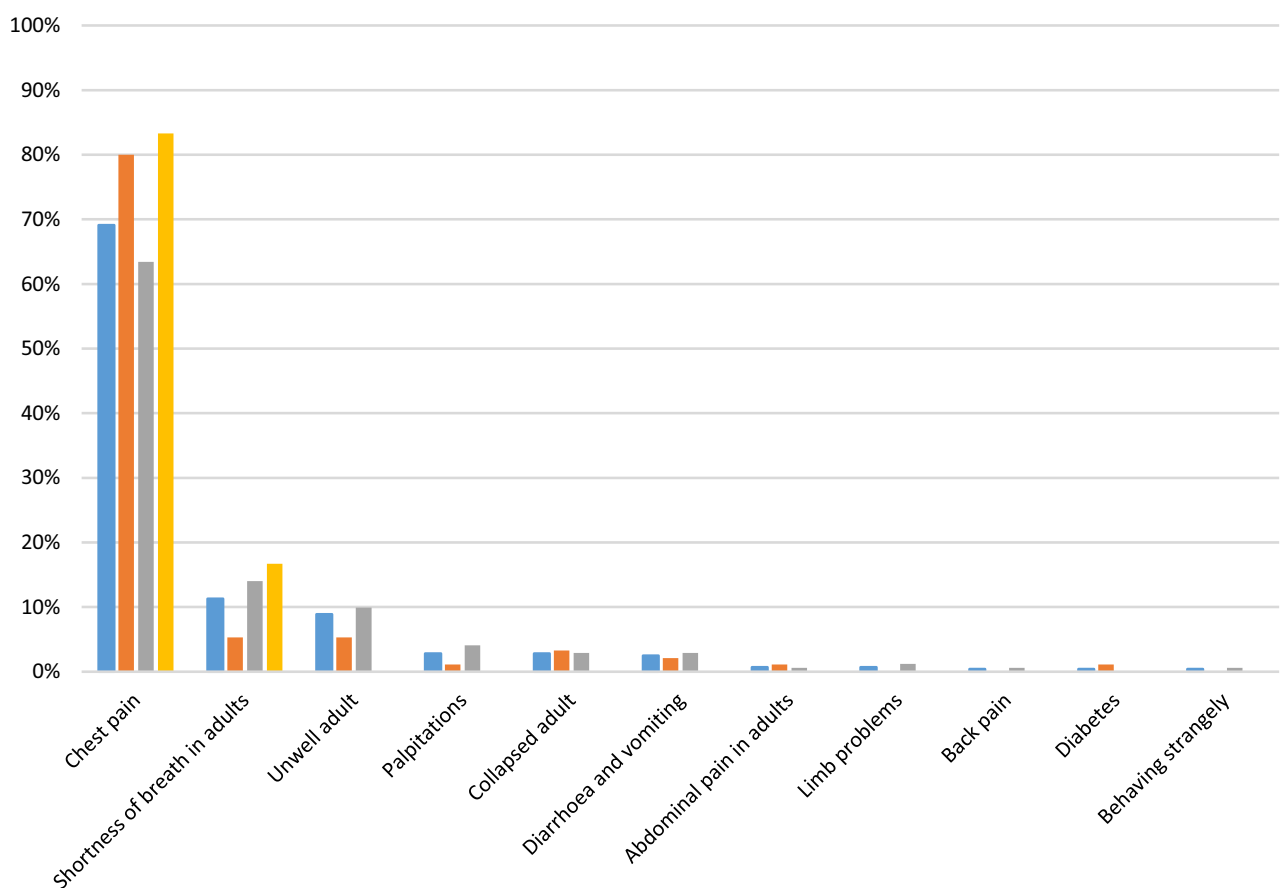

ues of creatinine kinase as a marker of infarct size for patients triaged as MTS 1-3 (MTS 1: $1707 \mathrm{U} / 1$, MTS 2: $891 \pm 1229 U / 1$, MTS 3: $889 \pm 1436 U / 1$, MTS 4: $660 \pm 1157 \mathrm{U} / \mathrm{l})$. However, we consider that maximum values of creatinine kinase were more influenced by the time from symptom onset to revascularization than by the assigned MTS levels.

Matias et al. demonstrated a comparable MTS level distribution to our study in a smaller study population of 114 patients with ACS (MTS level 1: 0.9\%, MTS level 2: $62.3 \%$, MTS level 3: $16.7 \%$, MTS level 4: 10.5\%, MTS level 5: 1.8\%) [7]. The relatively low proportion of patients classified as MTS level 1 in this and our study might be explained by direct admissions to medical intensive care units of hemodynamically and/or respiratory unstable patients bypassing the ED.

The sensitivity of the MTS for detecting high risk cardiac patients by nurses was reported to be $86.8 \%$ (95\% CI $78.4-92.3 \%)$ with a specificity of $72.4 \%(95 \%$ CI $61.4-81.2 \%$ ) [8]. A slightly higher sensitivity of 87.3\% (95\% CI 83.1-90.6\%) was reported for the MTS in assigning high priority (MTS level 1 and 2) to patients with ACS [9].

We did not observe a significant difference of mean MTS levels with respect to different types of ACS. Therefore, differentiation of STEMI from N-STEMI or UAP is from our experience not possible with the MTS, as the diagnosis of STEMI, N-STEMI and UAP is based on ECG criteria and troponin levels. However, it has been proven that the MTS is effective in patients with STEMI with typical symptoms with respect to first medical assessment within target time [10]. The observed higher mean MTS levels for female and patients older than 80 years may be explained by a presentation with other or atypical symptoms.
Chest pain was the most frequently used flow chart diagram in our study population ( $n=195,69.1 \%)$ and was even more frequently used in patients with STEMI $(n=76,80.0 \%$ ) (Fig. 3). Fewer patients with N-STEMI $(n=109,63.4 \%)$ complained about chest pain and shortness of breath was more frequently in patients with N-STEMI $(n=24,14.0 \%)$ compared to patients with STEMI $(n=5,5.3 \%)$. Other flow chart diagrams were less frequently used in our study population (unwell adult: $n=24,8.5 \%$; palpitations: $n=8$, $2.8 \%$; collapsed adult: $n=8,2.8 \%)$. Two hundred and twenty-seven patients $(80.5 \%)$ presented with typical symptoms of myocardial infarction (chest pain or shortness of breath). Therefore, we believe that the MTS is a valuable tool to detect patients with STEMI or N-STEMI early in the ED.

Remarkably, our study demonstrated that the majority of patients with cardiogenic shock had an onset of shock after triage in the ED. Therefore, we believe that patients with suspected ACS with typical symptoms, e.g. chest pain, shortness of breath, should be monitored on cardiac care units to detect deteriorating patients early, irrespective of the assigned MTS level. The in-hospital mortality of $2.5 \%$ was low. Three of these patients were classified as MTS level 2 and 3, respectively and one patient as MTS level 4. Several studies have shown that the MTS predicts hospital admission and mortality in an unselected all-comers study population [11-14].

No data were found in the literature for the emergency severity index and the Australasian triage scale with respect to acute coronary syndrome or myocardial infarction. A large study in Canada which included over 3000 patients with STEMI and NSTEMI demonstrated that half of the patients were given 
a low acuity triage score of 3,4 or 5 with the Canadian triage and acuity scale which was independently associated with substantial delays in ECG acquisition (median door-to-ECG time $12.0 \mathrm{~min}$ with a $4.4 \mathrm{~min}$ delay in median door-to-ECG time) and to reperfusion therapy (median door-to-needle time $40.0 \mathrm{~min}$ with a $15.1 \mathrm{~min}$ delay in median door-to-needle time). Therefore, the authors concluded that the quality of ED triage may have an important impact on acute myocardial infarction care [15].

\section{Limitations of this study}

However, we are well aware of the fact that our findings must be interpreted with caution. First, it is a single center experience. Second, the retrospective design can only be considered as hypothesis generating. Third, the number of patients is limited.

\section{Conclusion}

The majority of patients diagnosed with ACS, who presented to the ED and were triaged by the MTS, were classified as MTS levels 2 and 3 (very urgent to urgent assessment within 10 to 30 min [German version]/60 min [British version]). We observed significant lower MTS levels for males and patients younger than 80 years. As discrimination of different types of ACS is not possible with the MTS, we recommend that an ECG should be recorded in patients suspected for ACS immediately or at least within 10 min after first medical contact in the ED according to current guidelines, irrespective of the assigned MTS level in order to assure an early diagnosis of STEMI.

Funding Open access funding provided by Kepler Universitätsklinikum Linz.

Conflict of interest D. Kiblboeck, K. Steinrueck, C. Nitsche, W. Lang, J. Kellermair, H. Blessberger, C. Steinwender, and P. Siostrzonek declare that they have no competing interests.

Open Access This article is licensed under a Creative Commons Attribution 4.0 International License, which permits use, sharing, adaptation, distribution and reproduction in any medium or format, as long as you give appropriate credit to the original author(s) and the source, provide a link to the Creative Commons licence, and indicate if changes were made. The images or other third party material in this article are included in the article's Creative Commons licence, unless indicated otherwise in a credit line to the material. If material is not included in the article's Creative Commons licence and your intended use is not permitted by statutory regulation or exceeds the permitted use, you will need to obtain permission directly from the copyright holder. To view a copy of this licence, visit http://creativecommons.org/licenses/by/4.0/.

\section{References}

1. Fernandez JB, Ezquerra EA, Genover XB, O'Callaghan AC, Garriz II, Nacher JJ, et al. Chest pain units. Organization and protocol for the diagnosis of acute coronary syndromes. RevEsp Cardiol. 2002;55:143-54.

2. Amsterdam EA, Kirk JD, Bluemke DA, Diercks D, Farkouh ME, Garvey JL, et al. Testing of low-risk patients presenting to the emergency department with chest pain: a scientific statement from the American Heart Associaton. Circulation. 2010;122(17):1756-76.

3. Leite L, Baptista R, Leitao J, Cochicho J, Breda F, Elvas L, et al. Chest pain in the emergency department: risk stratification with Manchester Triage System and HEART score. BMC Cardiovasc Disord. 2015;15:48.

4. Mackway-Jones K, Marsden J, Windle J. Ersteinschaetzung inder Notaufnahme: DasManchesterTriageSystem. 3rded. Bern: Huber; 2011.

5. Roffi M, Patrono C, Collet JP, Mueller C, Valgimigli M, Andreotti F, et al. ESC Guidelines for the management of acute coronary syndromes in patients presenting without persistent ST-segment elevation: Task Force for the Management of Acute Coronary Syndromes in Patients Presenting without Persistent ST-Segment Elevation of the European Society of Cardiology (ESC). Eur HeartJ. 2016;37(3):267-315.

6. Gouvea VET, Reis MAM, Gouvea GM, Lima HN, Abuabara A. Evaluation of the Manchester Triage System in the acute coronary syndrome. Int J Cardiovasc Sci. 2008;28(2):107-13.

7. Matias C, Oliverira R, Duarte R, Bico P, Mendonca C, Nunu L, et al. The Manchester Triage System in acute coronary syndromes. Rev Port Cardiol. 2008;27(2):205-16.

8. Speake D, Teece S, Mackway-Jones K. Detecting high riskpatients with chest pain. Emerg Nurse. 2003;11(5):19-21.

9. Pinto D, LunetN, AzevedoA. Sensitivity and specificity of the Manchester Triage System for patients with acute coronary syndrome. Rev Port Cardiol. 2010;29(06):961-87.

10. Providencia R, Gomes PL, Barra S, Silva J, Seca L, Antunes A, et al. Importance of Manchester Triage in acute myocardial infarction: impact on prognosis. Emerg Med J. 2011;28(3):212-6.

11. Martins HM, Cuna LM, Freitas P. Is Manchester (MTS) more than a triage system? Astudy ofits association withmortality and admission to a large Portuguese hospital. Emerg Med J. 2009;26(3):183-6.

12. van der Wulp I, Schrijvers AJ, van Stel HF. Predicting admission and mortality with the Emergency Severity Index and the Manchester Triage System: a retrospective observational study. Emerg Med J.2009;26(7):506-9.

13. Azeredo TR, Guedes HM, Rebelo de Almeida RA, Chianca TC, Martins JC. Efficacy of the Manchester Triage System: a systematic review. Int Emerg Nurs. 2015;23(2):47-52.

14. Graeff I, Goldschmidt B, Glien P, Bogdanow M, Fimmers R, Hoeft A, et al. The German version of the Manchester Triage System and its quality criteria-first assessment of validity and reliability. PloS ONE. 2014;9(2):e88995.

15. Atzema CL, Austin PC, Tu JV, Schull MJ. Emergency department triage of acute myocardial infarction patients and the effect on outcomes. Ann Emerg Med. 2009;53(6):736-45.

Publisher's Note Springer Nature remains neutral with regard to jurisdictional claims in published maps and institutional affiliations. 